\title{
Suppression of the Epidermal Growth Factor-like Domain 7 and Inhibition of Migration and Epithelial-Mesenchymal Transition in Human Pancreatic Cancer PANC-1 Cells
}

\author{
Yun-Liang Wang ${ }^{1 \&}$, Feng-Lin Dong ${ }^{2 \&}$, Jian Yang ${ }^{1 \&}, \mathrm{Zhi} \mathrm{Li}^{3}$, Qiao-Ming Zhi ${ }^{1}$, Xin \\ Zhao ${ }^{1}$, Yong Yang ${ }^{1}$, De-Chun $\mathrm{Li}^{1}$, Xiao-Chun Shen ${ }^{4 *}$, Jin Zhou ${ }^{1 *}$
}

\begin{abstract}
Background: Epidermal growth factor-like domain multiple 7 (EGFL7), a secreted protein specifically expressed by endothelial cells during embryogenesis, recently was identified as a critical gene in tumor metastasis. Epithelial-mesenchymal transition (EMT) was found to be closely related with tumor progression. Accordingly, it is important to investigate the migration and EMT change after knock-down of EGFL7 gene expression in human pancreatic cancer cells. Materials and Methods: EGFL7 expression was firstly testified in 4 pancreatic cancer cell lines by real-time polymerase chain reaction (Real-time PCR) and western blot, and the highest expression of EGFL7 was found in PANC-1 cell line. Then, PANC-1 cells transfected with small interference RNA (siRNA) of EGFL7 using plasmid vector were named si-PANC-1, while transfected with negative control plasmid vector were called NC-PANC-1. Transwell assay was used to analyze the migration of PANC-1 cells. Real-time PCR and western blotting were used to detect the expression change of EGFL7 gene, EMT markers like E-Cadherin, N-Cadherin, Vimentin, Fibronectin and transcription factors like snail, slug in PANC-1, NCPANC-1, and si-PANC-1 cells, respectively. Results: After successful plasmid transfection, EGFL7 gene were dramatically knock-down by RNA interference in si-PANC-1 group. Meanwhile, migration ability decreased significantly, compared with PANC-1 and NC-PANC-1 group. Meanwhile, the expression of epithelial phenotype marker E-Cadherin increased and that of mesenchymal phenotype markers $\mathrm{N}$-Cadherin, Vimentin, Fibronectin dramatically decreased in si-PANC-1 group, indicating a reversion of EMT. Also, transcription factors snail and slug decreased significantly after RNA interference. Conclusions: Current study suggested that highly-expressed EGFL7 promotes migration of PANC-1 cells and acts through transcription factors snail and slug to induce EMT, and further study is needed to confirm this issue.
\end{abstract}

Keywords: Pancreatic cancer - epidermal growth factor-like domain multiple 7 - epithelial-mesenchymal transition

Asian Pac J Cancer Prev, 16 (9), 4065-4069

\section{Introduction}

Pancreatic carcinoma is the $4^{\text {th }}$ leading cause of cancerrelated death in the world (Chen et al., 2013; Liu et al., 2014; Siegel et al., 2015). It is reported that the overall 5 year survival rates to be below $5 \%$ (Vincent et al., 2011; Chen et al., 2013; Liu et al., 2014). The long-term survival of pancreatic carcinoma has not been improved within last two decades, despite widely-performed surgical resections. The dismal prognosis of pancreatic cancer is mainly due to its high metastatic potential, the late manifestation (Hackert and Buchler, 2013). Thus, understanding the molecular mechanisms of pancreatic cancer progression should be helpful to develop efficient treatments for the disease (Chang et al., 2012).

The developments, growth, and invasion of pancreatic cancer are driven by multiple genetic and epigenetic events. Epidermal growth factor-like domain multiple 7 (EGFL7) is a secreted protein that contains two EGFlike domains and is conserved across species (Nichol and Stuhlmann, 2012). It was initially regarded as an endothelial cell-specific gene and an important regulator in tubulogenesis during embryonic development (Fitch et al., 2004). However, recent studies have demonstrated that EGFL 7 expresses in several tumors and many cancer cell lines, and is associated with poor prognosis (Fan et al., 2013). Zhou et al reported that EGFL7 expression in pancreatic carcinoma tissues was significantly higher than that in non-tumor tissues, and high expression of EGFL7 in pancreatic carcinoma tissues was significantly associated with poor overall survival, accompanied by several conventional clinicopathological variables, such

${ }^{1}$ Department of General Surgery, ${ }^{2}$ Department of Ultrasonography, ${ }^{3}$ Department of Interventional Radiology, ${ }^{4}$ Department of Respiratory Medicine, the First Affiliated Hospital of Soochow University, Suzhou, Jiangsu, China \&Equal contributors *For correspondence: 13913500369@163.com; sxcsmile20008@163.com 
as lymph node metastasis, histological grade and gender (Zhou et al., 2014). However, further studies are needed to investigate the role and mechanisms of EGFL7 in the invasion of pancreatic carcinoma.

Epithelial-mesenchymal transition (EMT) has been found to be closely related with carcinoma progression, and acts as a major driver of tumor invasion (Thiery et al., 2009). In recent years, the targeting of EMTrelated pathways in human tumor cell lines and murine models revealed the crucial importance of EMT in the aggressiveness and invasion of pancreatic cancer (Kurahara et al., 2012). Luo et al reported that EGFL7 promotes tumor invasion by activating EMT through an EGFR-AKT-Snail signaling pathway in gastric cancer (Luo et al., 2014). Thus, we assumed that EGFL7 may also promote cancer invasion by inducing EMT in pancreatic cancer.

In this study, we assessed the biological behavior change in PANC-1 cells after EGFL7 RNA interference (RNAi), investigated the expression of epithelial, mesenchymal, and transcriptional markers and observed EGFL7 on triggering EMT progression in pancreatic cancer.

\section{Materials and Methods}

\section{Cell line and reagents}

Four human pancreatic cancer cell lines including Patu8988, PANC-1, BxPc-3, and Capan-1 were obtained from American Type Culture Collection(ATCC, Manassas, VA, USA) and cultured in Dulbecco's modified Eagle's medium(DMEM, Sigma-Aldrich, St. Louis, MO, USA) supplemented with $10 \%$ fetal bovine serum (FBS, Sijiqing biochemical, Hangzhou, China). Trizol reagent and M-MLV were purchased from Gibco BRL (Invitrogen, Carlsbad, CA, USA). Taq DNA polymerase, dNTPs, and DNA marker were obtained from Takara Bio Co (Dalian, China). Monoclonal mouse anti-human EGFL7, E-Cadherin, N-Cadherin, Vimentin, Fibronectin, Snail, and Slug were purchased from Santa Cruz Biotechnology (Santa Cruz, Paso Robles, CA, USA). MTT were purchased from Sigma-Aldrich (3050 Spruce St, St. Louis, MO USA).

\section{Cell culture and plasmid infection}

PANC-1 cells was maintained in DMEM supplemented with $100 \mathrm{ml} / \mathrm{LFBS}$, and incubated at $37^{\circ} \mathrm{C}$ in a humidified atmosphere containing $50 \mathrm{ml} / \mathrm{l} \mathrm{CO}_{2}$ in air. Cells were subcultured at $5 \times 10^{4}$ cells per well into six-well tissue culture plates overnight. Small hairpin RNA (shRNA) of human EGFL7 (GenBank, NM_016215) plasmid transfer vector encoding green fluorescent protein (GFP) sequence was constructed and transfected into PANC-1 cell line by Shanghai Genechem Co (Shanghai, China).

\section{Quantification by Real-time PCR}

Total RNA was collected by using the Trizol reagent following the manufacturer's instructions. The concentration and purity of the total RNA were detected with ultraviolet spectrophotometer and then reversely transcribed into cDNA with M-MLV. Quantitative real- time PCR assays were carried out using SYBR Green realtime PCR Master Mix and real-time PCR amplification equipment. Human actin was used as internal control. The PCR conditions consisted of 1 cycle at $95^{\circ} \mathrm{C}$ for $15 \mathrm{~s}$ followed by 45 cycles at $95^{\circ} \mathrm{C}$ for $5 \mathrm{~s}$ and at $60^{\circ} \mathrm{C}$ for $30 \mathrm{~s}$. The primer sequences were as follows: EGFL7 Forward, 5'-TGAATGCAGTGCTAGGAGGG-3'; Reverse, 5'-GCACACAGAGTGTACCGTCT-3'. E-Cadherin Forward, 5'-GTCTCTCTCACCACCTCCACAG-3'; Reverse, 5'-CTCGGACACTTCCACTCTCTTT-3'. $\mathrm{N}-\mathrm{C}$ a d h e r i n F o r w a r d, 5'-TGCTACTTTCCTTGCTTCTGAC-3'; Reverse, 5'-TAACACTTGAGGGGCATTGTC-3'. Vimentin Forward, 5'-GAAGAGAACTTTGCCGTTGAAG-3'; Reverse, 5'-GAAGGTGACGAGCCATTTC-3'. $\mathrm{F}$ i b r o n e c t i $n \quad F$ o $r$ w a $r$ d, 5'-AGGAAGCCGAGGTTTTAACTG-3'; Reverse, 5'-AGGACGCTCATAAGTGTCACC-3'. Snail Forward: 5'-TCGGAAGCCTAACTACAGCGA-3'; Reverse: 5'-AGATGAGCATTGGCAGCGAG-3'. Slug Forward: 5'-CGAACTGGACACACATACAGTG-3' Reverse: 5'-CTGAGGATCTCTGGTTGTGGT-3'. GAPDH Forward, 5'-ACAACTTTGGTATCGTGGAAGG-3'; Reverse, 5'-GCCATCACGCCACAGTTTC-3'.

\section{Western blot analysis}

Cells were washed twice and lysed on ice. After centrifugation, the supernatant were collected. Protein concentrations were determined by the Bio-Rad Dc protein assay system. Samples were then separated on $10 \%$ SDSPAGE and transferred onto PVDF membrane. Membranes were blocked and incubated with primary anti- EGFL7 antibody (1:100 dilution ), anti- $\beta$-actin antibody (1:200 dilution ) at $4{ }^{\circ} \mathrm{C}$ overnight. After 3 washes, the membranes underwent hybridization with a goat anti-mouse $\mathrm{IgG}$ conjugated with horseradish peroxidase (1:5000 dilution) for $2 \mathrm{~h}$ at room temperature. After further washing, reactive bands were visualized using ECLTM Western Blot Detection Reagents with exposure to X-ray film for 30-120 $\mathrm{s}$. The bands intensities were calculated by densitometric analysis using Image $\mathbf{J}$ software.

\section{Boyden chamber transwell assay}

We used modified Boyden chambers with filter inserts (pore size, $8 \mu \mathrm{m}$ ) coated with Matrigel in 24-well dishes. Approximately $2.5 \times 10^{4}$ cells in $100 \mu 1$ of complete DMEM medium were placed in the upper chamber, and $1 \mathrm{ml}$ of the same medium was placed in the lower chamber. After $24 \mathrm{~h}$ culture, cells were fixed in methanol for $15 \mathrm{~min}$ and then stained with $0.05 \%$ crystal violet in PBS for $15 \mathrm{~min}$. Cells on the upper side of the filters were removed with cottontipped swabs. Cells that had invaded into the lower side of the filter were then stained and microscopically observed and counted in 5 fields of view at $40 \times$ magnification. The area of each field equals to $0.3 \mathrm{~mm}^{2}$. The invasive activity of cancer cells was expressed as the mean number of cells that invaded to the lower side of the filter.

\section{Statistical analysis}

Each experiment was performed at least three times and found to be reproducible. Data were shown as mean 
\pm SD and the statistical significance of differences between the groups was determined by applying one-way analysis of variance (ANOVA), followed by Fisher's least significant difference. $P<0.05$ was considered statistically significant. These analyses were performed using SPSS 19.0 software.

\section{Results}

Screened EGFL7 expression in four cell lines

After the real-time PCR analysis, PANC-1 cells demonstrated the highest mRNA expression $(2.266 \pm 0.20)$, compared with other 3 cell lines $(P<0.05)$. Also in western blot test, we found the maximal protein expression in PANC-1 cells $(0.32 \pm 0.04)$, compared with other 3 cell lines $(P<0.05)$, similar results with PCR analysis. Accordingly, we choose PANC-1 cells as the target cell for the below RNAi research.

\section{Plasmid stably-infected PANC-1 Cell}

After successful infection with a plasmid vector carrying a green fluorescence protein(GFP) reporter gene, immunofluorescence showed that nearly more than $90 \%$ of GFP positive expression in si-PANC-1 and NC-PANC-1 groups (Figure 1A), indicating a high and stable transfection.

\section{Expression of EGFL7 after RNAi}

To determine the effect of the EGFL7 RNAi, real-time PCR and Western blot were performed to determine the mRNA and protein levels of EGFL7 after vector infection for $120 \mathrm{~h}$. As is shown in Figure 1B, the relative EGFL7 mRNA expression $(0.31 \pm 0.01, P<0.05)$ was significantly decreased in si-PANC-1 group compared with the NCPANC-1 $(0.93 \pm 0.05)$ and the PANC-1 group, but there were no significant differences between NC-PANC-1 group and PANC-1 group ( $P>0.05)$. Western blot results showed that an approximately $30 \mathrm{kDa}$ protein band was observed, EGFL7 protein was weakly expressed in the siPANC-1 group, but there were no significant differences

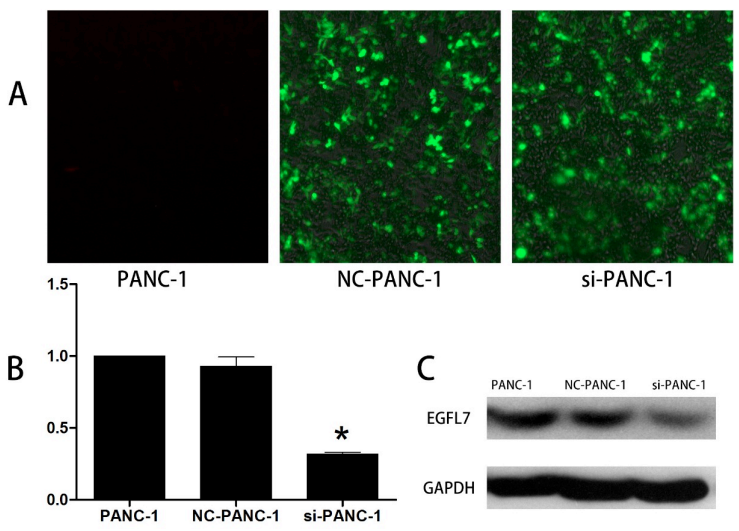

Figure 1. Expression of EGFL7 in PANC-1 Cells after RNAi. (A) Immunofluorescence for detection the efficiency of plasmid vector infection, and GFP was used as reporter gene. (B) The graph showed the expression of EGFL7 in si-PANC-1 was decreased significantly compared with PANC-1 and NC-PANC-1 by real-time PCR $(* \mathrm{P}<0.05)$. (C) Western blot confirmed that the protein level of EGFL7 in si-PANC-1 was also reduced
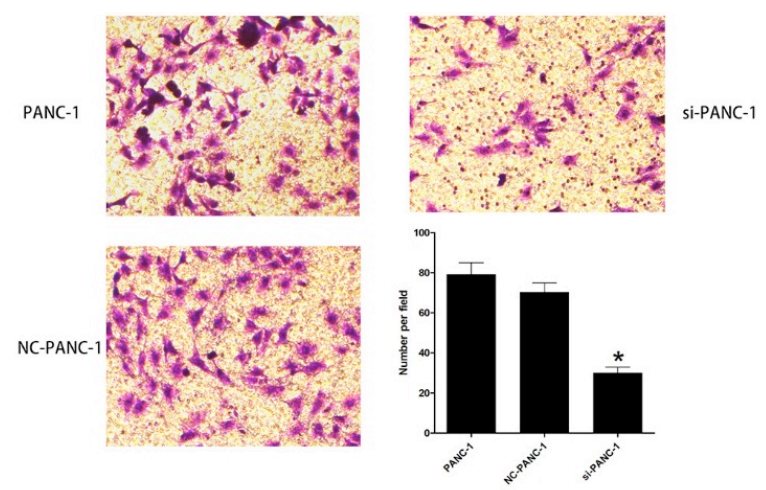

Figure 2. Migration Cells in 3 Groups Assessed by Transwell Assay. Representative photographs of migration ability with PANC-1, NC-PANC-1 and si-PANC-1 are shown above. Significantly fewer cells of si-PANC- 1 group passed through the Matrigel compared to PANC-1 and NC-PANC-1. $(* \mathrm{P}<0.05)$

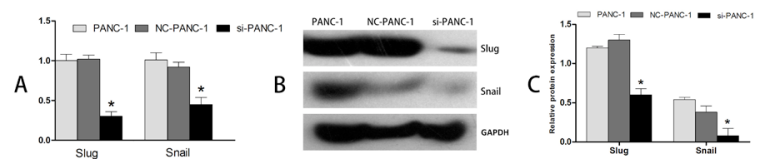

Figure 4. Expression of Slug and Snail in 3 Groups. (A) Lower Slug and Snail mRNA level was observed in si-PANC-1 cells. $(\mathbf{B}, \mathbf{C})$ The same result was observed by Western blot. $(* \mathrm{P}<0.05)$

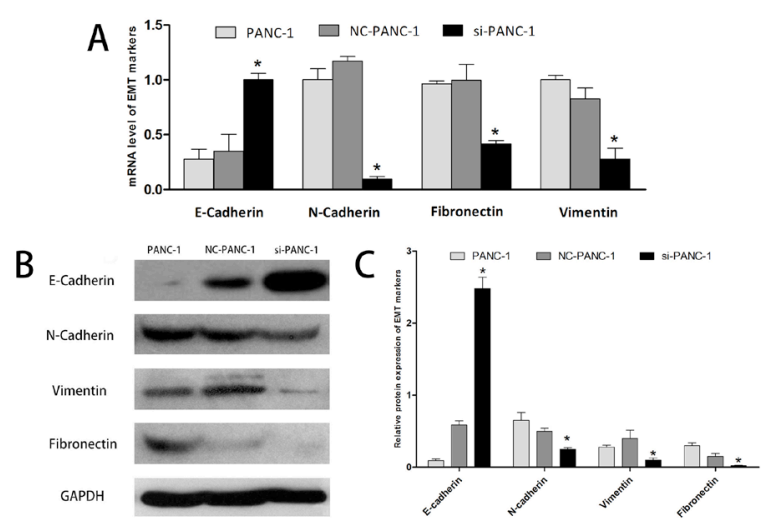

Figure 3. The Expression of E-Cadherin, N-Cadherin, Fibronectin and Vimentin in mRNA and Protein Level. (A) Higher E-cadherin mRNA levels and lower N-cadherin, fibronectin, vimentin mRNA levels were observed in si-PANC-1 cells. (B) Western blot analysis of E-cadherin, N-cadherin, fibronectin and vimentin protein in PANC-1, NC-PANC-1 and si-PANC-1 cells. (C) Higher E-cadherin protein and lower $\mathrm{N}$-cadherin, fibronectin, vimentin protein were observed in siPANC-1 cells. $(* \mathrm{P}<0.05)$

between NC-PANC-1 group and PANC-1 group (Figure 1C).

Invasion ability by transwell assay

Boyden chambers assay was used to investigate the invasive ability after the knockdown of EGFL7 in vitro. As is shown in Figure 2, the number of invaded cells in the si-PANC-1 group (30 \pm 2.5$)$ cells/well was dramatically decreased than that in the PANC-1 group and the NCPANC-1 group $(P<0.05)$. The metastatic response was effectively inhibited by the RNA interference, but there 
were no significant differences between the NC-PANC-1 group (70 \pm 4.0$)$ cells/well and the control group $(79 \pm 5.1)$ cells/well by counting the invasive cell number $(P>0.05)$.

\section{Effects of EGFL7 RNAi on Expression of EMT Markers in PANC-1 Cells}

To further explore whether the EGFL7 RNAi affects specific molecular marker of EMT in PANC-1 cells, we detected the expression of E-cadherin, $\mathrm{N}$-cadherin, vimentin, and fibronectin in PANC-1, NC-PANC-1, and siPANC-1 cells by real-time PCR and Western blot. Compared with PANC-1 and NC-PANC-1, the expression of E-cadherin in si-PANC-1 cells mRNA $(1.00 \pm 0.06, P<0.05)$ and protein levels $(2.50 \pm 0.16$, $P<0.05)$ significantly increased; in contrast, those of the mesenchymal cell markers $\mathrm{N}$-cadherin, fibronectin, and vimentin significantly decreased. No significant differences were found between PANC-1 and NC-PANC-1 cells (Figure 3).

\section{Effects of EGFL7 RNAi on expression of transcription factors in PANC-1 cells}

We tested the expression of the transcription factors snail and slug by real-time PCR and western blot to determine whether they are involved in EGFL7induced EMT in PANC-1 cells. The mRNA and protein expression of snail in si-PANC-1 cells were $(0.45 \pm 0.09)$ and $(0.05 \pm 0.004)$, respectively, while that of slug were $(0.31 \pm 0.06)$ and $(0.09 \pm 0.01)$, respectively. All these decreased significantly compared with that of $\mathrm{NC}$ PANC-1 group and control group $(P<0.05)$. No significant differences in expression of snail and slug were observed between NC-PANC-1 and PANC-1 cells $(P>0.05$, Figure 4).

\section{Discussion}

EGFL7, also known as vascular endothelial statin (VEstatin), was initially characterized as a gene specifically expressed by blood vessel endothelial cells in normal organs during development and in adult(Soncin et al., 2003; Parker et al., 2004; Nichol and Stuhlmann, 2012). However, recent studies have reported elevated expression of EGFL7 in several tumors and cancer cell lines, including pancreatic cancer (Zhou et al., 2014), kidney tumors (Xu et al., 2014), malignant gliomas(Huang et al., 2010), hepatocellular carcinomas (Wu et al., 2009; Li et al., 2015), and colon cancers (Diaz et al., 2008). Zhou et al reported that high EGFL7 expression in tumor tissues was significantly associated with poor overall survival, and was identified as an independent marker for long-term outcome of pancreatic carcinoma (Zhou et al., 2014). All these observations strongly suggested a role for EGFL7 in the migration of pancreatic carcinoma.

In this study, we firstly screened 4 pancreatic cancer cell lines and successfully knocked down EGFL7 in PANC-1 cells by RNAi using a plasmid expression vector. After that, invasive potential was assessed by the Matrigel invasion assay. Significantly fewer si-PANC-1 cells traveled through the transwell filter compared to PANC-1 and NC-PANC-1 groups. These findings indicate that EGFL7 under-expression inhibited pancreatic cancer cells invasion. Interestingly, the effect of EGFL7 on pancreatic cancer cell is in the similar manner to its effect on endothelial cells: the loss of EGFL7 inhibits the migration of endothelial cells, but plays no effect on the proliferation of ECs (Nichol and Stuhlmann, 2012).

EMT is a biologic process that allows a polarized epithelial cell, which normally interacts with basement membrane via its basal surface, to undergo multiple biochemical changes. Inside this process, EMT enable to assume a mesenchymal cell phenotype, which includes enhanced migratory capacity, invasiveness, elevated resistance to apoptosis, and greatly increased production of extracellular matrix components. The completion of an EMT is signaled by the degradation of underlying basement membrane and the formation of a mesenchymal cell that can migrate away from the epithelial layer in which it originated (Techasen et al., 2012; Liu et al., 2015).

The mechanism of EGFL7 promoting cancer cell migration is unclear. Several studies have shown that EGF promotes cancer cell migration with activation of EMT (Luo et al., 2014). And EGFL7 contains two EGF-like domains in its protein organization. This suggests that EGFL7 may be a promoter in cancer cell migration. Luo et al reported that EGFL 7 promotes tumor invasion by activating EMT through an EGFR-AKT-Snail signaling pathway in gastric cancer (Luo et al., 2014).

In this study, the expression of Vimentin, N-cadherin, Fibronectin are found to be decreased in si-PANC-1 group compared with PANC-1 and NC-PANC-1 groups; and the expression of E-cadherin is found to be increased in si-PANC-1 group. These findings indicate that inhibiting EGFL7 expression can reverse EMT in pancreatic cancer cells. The idea that EMT has a role in cancer has also benefited from its link with the loss of E-cadherin expression. Key structures involved in maintaining this adhesiveness between cells include adherens junctions, tight junctions and gap junctions. One of the strongest and most studied regulators of adhesion is E-cadherin, a member of the cadherin family of proteins. E-cadherin, together with associated catenins, plays a key role in maintaining cell-cell adhesion, but which are lost or disturbed in cancer cells, mainly due to the loss of E-cadherin in cancer cells. Hence, reduced cell-cell adhesion after down-regulation of E-cadherin enhances the potential for metastatic dissemination of cancer cells (Beuran et al., 2015).

Next, we found decreased expression in transcription factor snail and slug after RNAi. Zinc finger protein snail and slug are proteins encoded by the snail gene. Snail is a family of transcription factors that promote the repression of the adhesion molecule E-cadherin to regulate EMT during embryonic development (Batlle et al., 2000). Our results suggested that down-regulation with EGFL7 can inhibit EMT via snail and slug transcriptional process.

This study had some limitations though the research done was carefully designed and performed. Firstly, the study indicated that knockdown of EGFL7 expression can inhibit invasion and EMT of pancreatic cancer PANC-1 cells, however, the relation of EGFL7 overexpression with invasion and EMT has not yet been proven. Secondly, 
the signaling pathway underlying the effects of EGFL7 on EMT in pancreatic cancer cells have not yet been explored clearly.

In conclusion, EGFL7 is an important regulator of pancreatic cancer cells migration. Blockade of EGFL7 by RNAi may suppress the migration of pancreatic cancer cells by reversing EMT. EGFL7 may be a useful target for gene therapy in pancreatic cancer. Meanwhile, the role of EGFL7 in pancreatic cancer, especially its biological behavior, needs our further study.

\section{Acknowledgements}

This study was supported by National Science Foundation of China (No.81302148) \& (No.81302146), the Science and Technology Bureau and Sanitary Bureau of Suzhou (KJXW2011008) \& (KJXW2013003), Soochow University (SDY2013A26), Natural Science Research Foundation of Colleges and Universities in Jiangsu Province (13KJB320019) \& (13KJB320018).

\section{References}

Batlle E, Sancho E, Franci C, et al (2000). The transcription factor snail is a repressor of E-cadherin gene expression in epithelial tumour cells. Nat Cell Biol, 2, 84-9.

Beuran M, Negoi I, Paun S, et al (2015). The epithelial to mesenchymal transition in pancreatic cancer: A systematic review. Pancreatology, [Epub ahead of print].

Chang ZG, Wei JM, Qin CF, et al (2012). Suppression of the epidermal growth factor receptor inhibits epithelialmesenchymal transition in human pancreatic cancer PANC-1 cells. Dig Dis Sci, 57, 1181-9.

Chen WQ, Liang D, Zhang SW, et al (2013). Pancreatic cancer incidence and mortality patterns in china, 2009. Asian Pac J Cancer Prev, 14, 7321-4.

Diaz R, Silva J, Garcia JM, et al (2008). Deregulated expression of miR-106a predicts survival in human colon cancer patients. Genes Chromosomes Cancer, 47, 794-802.

Fan C, Yang LY, Wu F, et al (2013). The expression of Egfl7 in human normal tissues and epithelial tumors. Int J Biol Markers, 28, 71-83.

Fitch MJ, Campagnolo L, Kuhnert F, et al (2004). Egfl7, a novel epidermal growth factor-domain gene expressed in endothelial cells. Dev Dyn, 230, 316-24.

Hackert T, Buchler MW (2013). Pancreatic cancer: advances in treatment, results and limitations. Dig Dis, 31, 51-6.

Huang CH, Li XJ, Zhou YZ, et al (2010). Expression and clinical significance of EGFL7 in malignant glioma. J Cancer Res Clin Oncol, 136, 1737-43.

Kurahara H, Takao S, Maemura K, et al (2012). Epithelialmesenchymal transition and mesenchymal-epithelial transition via regulation of ZEB-1 and ZEB-2 expression in pancreatic cancer. $J$ Surg Oncol, 105, 655-61.

Li Z, Ni CF, Zhou J, et al (2015). Expression of epidermal growth factor-like domain 7 is increased by transcatheter arterial embolization of liver tumors. Asian Pac J Cancer Prev, 16, 1191-6.

Liu Y, Zhou YD, Xiao YL, et al (2015). Cyr61/CCN1 Overexpression Induces Epithelial-Mesenchymal Transition Leading to Laryngeal Tumor Invasion and Metastasis and Poor Prognosis. Asian Pac J Cancer Prev, 16, 2659-64.

Liu SZ, Chen WQ, Wang N, et al(2014).Dietary factors and risk of pancreatic cancer: a multi-centre case-control study in China. Asian Pac J Cancer Prev, 15, 7947-50.
Luo BH, Xiong F, Wang JP, et al (2014). Epidermal growth factor-like domain-containing protein 7 (EGFL7) enhances EGF receptor-AKT signaling, epithelial-mesenchymal transition, and metastasis of gastric cancer cells. PLoS One, 9,99922.

Nichol D, Stuhlmann H (2012). EGFL7: a unique angiogenic signaling factor in vascular development and disease. Blood, 119, 1345-52.

Parker LH, Schmidt M, Jin SW, et al (2004). The endothelialcell-derived secreted factor Egfl7 regulates vascular tube formation. Nature, 428, 754-8.

Soncin F, Mattot V, Lionneton F, et al (2003). VE-statin, an endothelial repressor of smooth muscle cell migration. Embo $J, 22,5700-11$.

Techasen A, Loilome W, Namwat N, et al (2012). Cytokines released from activated human macrophages induce epithelial mesenchymal transition markers of cholangiocarcinoma cells. Asian Pac J Cancer Prev, 13, 115-8.

Thiery JP, Acloque H, Huang RYJ, et al (2009). EpithelialMesenchymal Transitions in Development and Disease. Cell, 139, 871-90.

Wu F, Yang LY, Li YF, et al (2009). Novel role for epidermal growth factor-like domain 7 in metastasis of human hepatocellular carcinoma. Hepatol, 50, 1839-50.

Xu HF, Chen L, Liu XD, et al (2014). Targeting EGFL7 expression through RNA interference suppresses renal cell carcinoma growth by inhibiting angiogenesis. Asian Pac J Cancer Prev, 15, 3045-50.

Zhou L, Li J, Zhao Y, et al (2014). Prognostic significance of epidermal growth factor-like domain 7 in pancreatic cancer. Hepatobiliary Pancreat Dis Int, 13, 523-8. 